\title{
Entropy and Channel Capacity in the Regenerative Setup with Applications to Markov Channels.
}

\author{
Vinod Sharma \\ Department of ECE \\ Indian Institute of Science \\ Bangalore, 560012, India \\ e-mail: vinodQece.iisc.ernet.in
}

\author{
S. K. Singh \\ Department of ECE \\ Indian Institute of Science \\ Bangalore, 560012, India \\ shail@pal.ece.iisc.ernet.in
}

\section{Application to Markov Channels}

Consider one user transmitting to another via a Markov fading channel. Let $\left(Z_{n}, S_{n}\right)$ be a countable, irreducible, aperiodic, ergodic Markov chain. Intervisit time to a particular state will represent a regeneration epoch. $S_{n}$ is the channel state. Given $Z_{n}, X_{n}$ is independent of everything else. Unlike in [3], our model allows inter-symbol interference.

Following [3], define $\rho_{n}(s, z)=P\left(S_{n}=s, Z_{n}=z \mid Y^{n-1}\right)$ and $\pi_{n}(s, z)=P\left(S_{n}=s, Z_{n}=z \mid Y_{1}^{n-1}, X_{1}^{n-1}\right)$. The proofs in [3] do not hold for Markov inputs in general. Our key observation is that $\left\{\pi_{n}\right\}$ and $\left\{\rho_{n}\right\}$ are regenerative sequences. The regeneration epochs of $\left\{\rho_{n}\right\}$ and $\left\{\pi_{n}\right\}$ are same as that of $\left\{\left(Z_{n}, S_{n}\right)\right\}$. Therefore, $\pi_{n} \rightarrow \pi$ and $\rho_{n} \rightarrow \rho$ converge in total variation to their unique distributions.

Next we provide recursive formulae for $I\left(X_{1}^{n}, Y_{1}^{n}\right)$. We have shown that

$I\left(X_{1}^{n}, Y_{1}^{n}\right)=\sum_{i=1}^{n}\left[-E\left[\log \left(\sum_{s, z} \sum_{x} P\left(Y_{i} \mid S_{i}=s, X_{i}=x\right)\right.\right.\right.$

$\left.\left.P\left(X_{i}=x \mid Z_{i}=z\right) \rho_{i}(s, z)\right)\right]+E\left[\log \left(\sum_{s, z} P\left(Y_{i} \mid X_{i}, S_{i}=s\right)\right.\right.$

$\left.\left.\left.\frac{P\left(X_{i} \mid S_{i}=s, Z_{i}=z\right) \pi_{i}(s, z)}{\sum_{s^{\prime}, z^{\prime}} P\left(X_{i} \mid S_{i}=s^{\prime}, Z_{i}=z^{\prime}\right) \pi_{i}\left(s^{\prime}, z^{\prime}\right)}\right)\right]\right]$.

We have obtained recursive formulae for $\rho_{n}$ and $\pi_{n}$ as in [3].

Now as in [3], we can obtain the limit of $I(X, Y) / n$. However unlike for the iid inputs, computing these distributions for Markov inputs is extremely complicated. Therefore, we consider another algorithm. Observe that, from our results $\left[\log P\left(Y_{1}^{n} \mid X_{1}^{n}\right) / P\left(X_{1}^{n}\right) / n\right] \rightarrow I(X, Y)$ a.s. and hence we can obtain recursive algorithms to compute an aproximation for the limit from formulae for $\rho_{n}$ and $\pi_{n}$.

We obtain a lower bound on channel capacity using the above algorithm, by calculating $\sup _{P(X)} I(X, Y)$ by restricting the supremum to the set of (hidden) Markov chain inputs. This lower bound is obviously tighter than $I_{i i d}$ obtained in [3]. As in [3], to compute this bound via optimization algorithms, it helps to know if $\lim _{n \rightarrow \infty} I\left(X_{1}^{n}, Y_{1}^{n}\right) / n$ is a continuous function of the distribution of $\left\{Z_{n}\right\}$. We have proved it, using the regenerative setup for finite state spaces.

The decision feedback decoder designed in the section VI of [3] can be directly extended to our setup. We have demonstrated the utility of our algorithm by applying on some Markov channels.

\section{REFERENCES}

[1] S. Asmussen, Applied Probability and Queues. Wiley. N.Y., 1987

[2] E. Biglieri, J. Proakis and S. Shamai, Fading Channels: Information theoretic and communication aspects, IEEE Transactions on Information Theory, Vol. 44, 1998, 2619-2692.

[3] A. J. Goldsmith and P. P. Varaiya, Capacity, mutual information and coding for finite state Markov channels, IEEE Transaction Information Theory, Vol. 42, 1996, $868-886$. 